\title{
Levantamento da fauna entomológica no Estado do Paraná. IV. Sphingidae (Lepidoptera). Diversidade alfa e estrutura de comunidade ${ }^{1}$
}

\author{
Renato C. Marinoni ${ }^{2}$ \\ Renato R.C. Dutra ${ }^{3}$ \\ Olaf H.H. Mielke ${ }^{2}$
}

\begin{abstract}
Survey of the entomological fauna in Paraná State. IV. Sphingidae (Lepidoptera). Alpha diversity and community structure. In addition to the Survey of the Entomological Fauna in Paraná State, the Sphingidae was studied in its ecological and faunistic aspects. The material was collected using one light trap installed in eight different sites of the State (Antonina, São José dos Pinhais, Colombo, Ponta Grossa, Telêmaco Borba, Jundiaí do Sul, Guarapuava, and Fênix), from October 1986 to September 1987. A total of 813 specimens and 55 species was captured. The highest values of diversity (Brillouin and Shannon) was observed in São José dos Pinhais and Jundiai do Sul; the highest values of evenness (Berger \& Parker, and Simpson) in São José dos Pinhais and Antonina. Besides using ecological indices, the data were also compared by Clustering Analysis and Linear Correlation Coefficient. The results were compared with meteorological and floristic conditions in the eight localities, and with the results of others Sphingidae surveys in the Neotropical region. KEY WORDS. Sphingidae, diversity, community structure, Paraná State
\end{abstract}

Com os esfingídeos são três as Famílias de Lepidoptera estudadas através do projeto "Levantamento da Fauna Entomológica no Estado do Paraná - PROFAUPAR" (MARINONI \& DUTRA 1996; MARINONI et al. 1997). Os esfingídeos serviram como base de estudos faunísticos em vários trabalhos, como os de YoUNG (1972) e SEIFERT (1974) que estudaram a fauna da Costa Rica, na América Central; CoELHO et al. (1979) trabalharam com a fauna de uma localidade do Estado de São Paulo; e FERREIRA et al. (1986) em localidade do Estado de Minas Gerais. LAROCA \& MielKe (1975) e LAROCA et al. (1989) trataram do estudo da fauna de Sphingidae da Serra do Mar, no Estado do Paraná, região onde também se localiza um dos pontos onde houve coleta pelo PROFAUPAR, São José dos Pinhais.

No "checklist" dos Sphingidae Neotropicais, recentemente publicado por CARCASSON \& HEPPNER (1996), foram listadas 408 espécies, sendo que 94 foram referidas para o Brasil, baseados apenas na indicação da localidade-tipo.

O presente trabalho contém estudos de diversidade alfa e de estrutura de comunidade de Sphingidae, baseados na coleta de 55 espécies e 813 indivíduos em oito localidades do Estado do Paraná.

1) Contribuição número 1165 do Departamento de Zoologia, Universidade Federal do Paraná.

2) Departamento de Zoologia, Universidade Federal do Paraná. Caixa Postal 19020, 81531-990 Curitiba, Paraná, Brasil.

3) Escola Técnica, Universidade Federal do Paraná. Rua Alcides Arcoverde 1225, 81520-260

Curitiba, Paraná, Brasil. 


\section{MATERIAL E MÉTODOS}

Os Sphingidae, na forma adulta, constituem um grupo de atividade predominantemente noturna, com espécies do gênero Aellopos, Hübner [1819] voando durante o dia (BIEZANKo 1948). Apesar deste autor ter indicado que estes lepidópteros apresentariam vôo crepuscular (com atividade também ao alvorecer) é possível constatar, pelos estudos do hábito de vôo de 22 espécies, feitos por SEIFERT (1974), em Turrialba, Costa Rica, que onze delas foram ativas durante toda a noite, seis em parte da noite e apenas cinco espécies com hábitos crepusculares (porém, pelo baixo número de observações, sem significância estatística).

Houve certa dificuldade na identificação de algumas espécies dos gêneros Manduca Hübner, [1807] e Nyceryx Boisduval, [1875]. No primeiro gênero algumas espécies foram arroladas a um grupo de espécies que inclui a espécie Manduca diffissa e outras a um grupo que inclui a espécie Manduca pellenia. No segundo gênero, há duas espécies - Nyceryx alophus e Nyceryx continua-com características muito semelhantes, e que, devido ao grande número de exemplares coletados, mostraram uma variação que poderá indicar serem sinônimas. Como esta sinonimização demanda estudos morfológicos e comparação ao material-tipo, assumiu-se que os exemplares coletados pertencem à Nyceryx continua, espécie mais antiga.

O material foi coletado em Antonina, São José dos Pinhais, Colombo, Ponta Grossa, Telêmaco Borba, Jundiaí do Sul, Guarapuava e Fênix, através de armadilha luminosa, durante 13 novilúnios, de outubro de 1986 a setembro de 1987; os dados sobre as localidades e o método e número de coletas foram apresentados em MARINONI \& DUTRA $(1991,1996)$.

\section{Análise dos dados meteorológicos e faunísticos}

A metodologia aplicada para estas análises já foram explicitadas em trabalhos anteriores (MARINONI \& DUTRA 1991, 1996; MARINONI et al. 1997). As referências às matrizes de dados utilizadas e as indicações dos métodos aplicados nas análises numéricas - análise de agrupamento - são feitas quando da apresentação dos resultados. Para as análises numéricas foi utilizado o programa NTSYS-PC, versão 1.8 , elaborado por ROHLF (1989).

\section{Índices de diversidade, dominância e uniformidade}

Foram utilizados os índices: 1) de riqueza (variedade) de espécies (= $\mathrm{S}) ; 2$ ) de Shannon (H'); 3) de Brillouin (HB); 4) $\alpha$ (alpha) de Williams; 5) de dominância de Berger \& Parker (BP); 6) de uniformidade de Shannon (H’E); 7) de uniformidade de Berger \& Parker (UBP); 8) de uniformidade de Simpson (1/D). As discussões sobre a aplicação dos diferentes índices foram feitas em MARINONI \& DUTRA (1996) e MARINONI et al. (1997).

\section{Material-testemunha}

O material encontra-se depositado na Coleção Entomológica Pe. Jesus S. Moure, Departamento de Zoologia, Universidade Federal do Paraná. 


\section{RESULTADOS E DISCUSSÃO}

NúMERO DE EXEMPLARES E dE ESPÉCIES DE SPHINGIDAE (Figs 1-9; Tabs I-IV)

Foram coletados 813 exemplares. São José dos Pinhais foi a localidade onde houve a maior abundância (486 exemplares), seguida de Jundiaí do Sul (94) e Guarapuava (68). O local de menor abundância foi Colombo com apenas 21 exemplares.

Foram capturadas 55 espécies. São José dos Pinhais apresentou o maior número de espécies (38), seguida de Jundiaí do Sul (23), Antonina e Ponta Grossa (15 espécies cada). As localidades com menor número de espécies foram Fênix e Telêmaco Borba (11) e Colombo (10). Dez espécies foram encontradas exclusivamente em São José dos Pinhais; 3 em Jundiaí do Sul; e 1 em Fênix, em Antonina, em Telêmaco Borba e em Ponta Grossa. Em Colombo e Guarapuava não houve captura de espécies exclusivas.

A análise dos dados com número acumulado de espécies capturadas, a cada novilúnio (Fig. 1; Tab. IV), indica que ao terceiro novilúnio já se somavam $71 \%$ do total capturado (39/55), superior, mas muito próximo, aos percentuais encontrados para Ctenuchidae (66\%) (MARINONI \& DUTRA 1996) e Saturniidae (65\%) (MARINONI et al. 1997). Uma análise de correlação linear, entre os vetores definidos pelos valores de acumulação do número de espécies coletadas de cada família, apresentou coeficientes altos e quase idênticos: $r=0,980$ entre Sphingidae e Ctenuchidae; 0,979 entre Sphingidae e Saturniidae e 0,981 entre Ctenuchidae e Saturniidae. Há uma clara indicação que os valores proporcionais de captura são semelhantes, apesar dos valores parciais e totais acumulados de novas espécies capturadas a cada novilúnio serem bastante distintos entre as famílias ( 94 espécies de Ctenuchidae; 83 espécies de Saturniidae e 55 de Sphingidae).

Dentre as duas espécies dominantes de cada local (Tab. III) destacam-se: Adhemarius gannascus em 7 localidades; Nyceryx continua em 3; Adhemarius eurysthenes, 2; Enyo gorgon, 1 ; Xylophanes tersa, 1; Manduca rustica, 1 e Erinnyis alope, 1 . As seis espécies dominantes, na soma de todas as oito localidades, foram: Adhemarius gannascus (156 exemplares), Nyceryx continua(92), Adhemarius eurysthenes (83), Erinnyis ello (55), Xylophanes tersa (50) e X.indistincta (33).

Apenas Adhemarius gannascus foi encontrada em todas as oito localidades e foi a mais abundante em seis delas: Guarapuava, Fênix, Ponta Grossa, Telêmaco Borba, Jundiaí do Sul, Antonina, e a segunda e terceira mais abundante em Colombo e São José dos Pinhais, respectivamente. Nestas localidades as espécies mais abundantes foram: Xylophanes tersa e Nyceryx continua, respectivamente.

Adhemarius gannascus, nos locais onde ocorreu de maneira mais abundante, como em São José dos Pinhais, Guarapuava e Jundiaí do Sul, foi capturada em quase todos os novilúnios.

$\mathrm{Na}$ análise dos valores de captura das espécies mais abundantes, por localidade, pode ser destacado:

Em São José dos Pinhais, com três picos de maior abundância, foram observadas as seguintes espécies: Nyceryx continua, em dezembro ( $1^{\circ}$ novilúnio) (9 exemplares), em fevereiro (19 exemplares) e outro em setembro (22); Adhemarius 


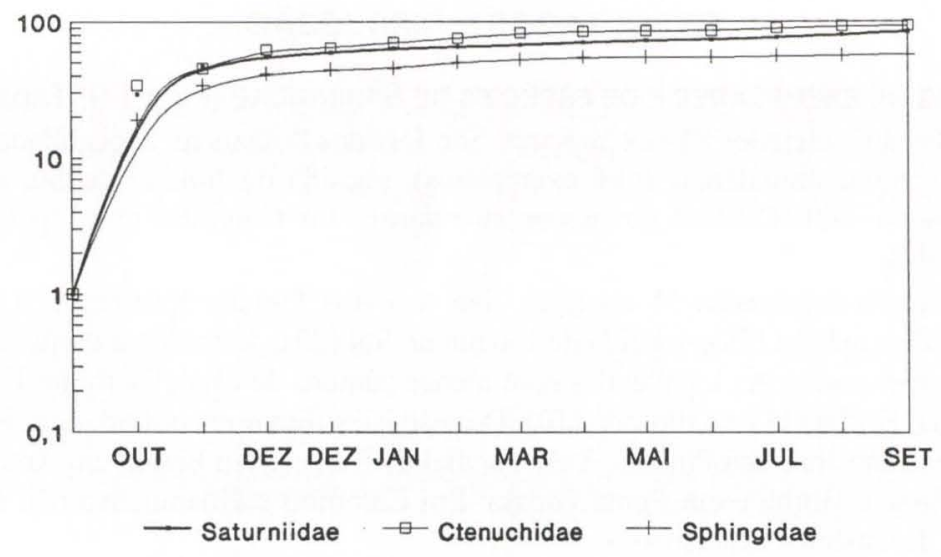

Fig. 1. Gráfico com a curva do número acumulado de diferentes espécies de Sphingidae capturadas a cada novilúnio; na ordenada, os logaritmos dos valores acumulados $(n+1)$.

Tabela I. Número total de exemplares de Sphingidae, por localidade, capturados durante 13 novilúnios, de outubro de 1986 a setembro de 1987.

\begin{tabular}{|c|c|c|c|c|c|c|c|c|c|c|c|c|c|c|}
\hline Novilúnio & 1 & 2 & 3 & 4 & 5 & 6 & 7 & 8 & 9 & 10 & 11 & 12 & 13 & \multirow{2}{*}{ Total } \\
\hline Localidade / Data & $03 / x$ & $02 / X I$ & $01 / X I I$ & $31 / X 11$ & $29 / 1$ & $27 / I I$ & $29 / I I I$ & $27 / \mathrm{IV}$ & $27 N$ & $26 \mathrm{NI}$ & $25 \mathrm{NII}$ & $24 / \mathrm{VIII}$ & $23 / 1 X$ & \\
\hline Antonina & 1 & 1 & 3 & 1 & 2 & 4 & 5 & 0 & 1 & 0 & 4 & 2 & 0 & 24 \\
\hline São José dos Pinhais & 4 & 20 & 80 & 61 & 58 & 85 & 92 & 9 & 4 & 0 & 29 & 11 & 33 & 486 \\
\hline Colombo & 0 & 8 & 0 & 2 & 3 & 4 & 0 & 1 & 0 & 0 & 1 & 1 & 1 & 21 \\
\hline Ponta Grossa & 5 & 3 & 4 & 4 & 6 & 7 & 10 & 6 & 2 & 0 & 7 & 3 & 4 & 61 \\
\hline Telémaco Borba & 3 & 5 & 2 & 3 & 3 & 0 & 3 & 1 & 3 & 0 & 0 & 1 & 3 & 27 \\
\hline Jundiai do Sul & 7 & 12 & 17 & 8 & 22 & 8 & 3 & 8 & 0 & 0 & 2 & 5 & 2 & 94 \\
\hline Guarapuava & 3 & 19 & 2 & 9 & 8 & 9 & 8 & 7 & 0 & 0 & 2 & 0 & 1 & 68 \\
\hline Fênix & 9 & 4 & 7 & 0 & 1 & 0 & 2 & 3 & 0 & 0 & 1 & 1 & 4 & 32 \\
\hline Total & 32 & 72 & 115 & 88 & 103 & 117 & 123 & 35 & 10 & 0 & 46 & 24 & 48 & 813 \\
\hline
\end{tabular}

eurysthenes, um em dezembro ( $2^{\circ}$ novilúnio) (17 exemplares), um em março (17), e outro em julho (6); Adhemarius gannascus, em dezembro ( $\left.1^{\circ}\right)$ (5 exemplares), em fevereiro (9) e julho (10). Com dois picos de maior abundância, as espécies: Manduca florestan, em dezembro $\left(1^{\circ}\right)$ (8 exemplares) e em janeiro (7); Xylophanes indistincta, em dezembro $\left(1^{\circ}\right)$ (5 exemplares) e fevereiro (13). Com apenas um pico de maior abundância, as espécies Erinnys ello, em dezembro $\left(1^{\circ}\right)$ (15 exemplares) e Xylophanes tersa, em março (28 exemplares).

Em Ponta Grossa, Adhemarius gannascus apresentou dois picos de maior captura, em janeiro (4 exemplares) e em julho (7).

Em Guarapuava, Adhemarius gannascus teve apenas um pico de maior abundância, em dezembro $\left(2^{\circ}\right)$ com oito exemplares.

Em Jundiaí do Sul, Adhemarius gannascus apresentou apenas um valor mais destacado de exemplares capturados, em janeiro; uma segunda espécie de abundância mais significativa no local, foi Manduca rustica, com picos em novembro (6) e dezembro $\left(2^{\circ}\right)(5)$.

Revta bras. Zool. 16 (Supl. 2): 223 - 240, 1999 
Tabela II. Espécies de Sphingidae capturadas em oito localidades do Estado do Paraná, durante treze novilúnios, no período de outubro de 1986 a setembro de 1987. (AN) Antonina; (SJ) São José dos Pinhais; (CO) Colombo; (PG) Ponta Grossa; (TB) Telêmaco Borba; (JS) Jundiai do Sul; (GU) Guarapuava; (FE) Fênix.

\begin{tabular}{|c|c|c|c|c|c|c|c|c|c|}
\hline Espécies / Localidades & AN & SJ & $\mathrm{CO}$ & $P G$ & TB & JS & GU & $\mathrm{FE}$ & Total \\
\hline Adhemarius eurysthenes (R. Felder, 1874) & 2 & 69 & 0 & 10 & 2 & 0 & 0 & 0 & 83 \\
\hline Adhemarius gannascus (Stoll, 1790) & 4 & 49 & 3 & 22 & 9 & 22 & 34 & 13 & 156 \\
\hline Agrius cingulatus (Fabricius, 1775) & 1 & 12 & 1 & 0 & 0 & 0 & 0 & 1 & 15 \\
\hline Callionima inuus (Rothschild \& Jordan, 1903) & 1 & 0 & 0 & 0 & 0 & 1 & 0 & 0 & 2 \\
\hline Callionima nomius (Walker, 1856) & 0 & 1 & 0 & 0 & 0 & 0 & 0 & 0 & 1 \\
\hline Callionima parce (Fabricius, 1775) & 0 & 1 & 0 & 1 & 0 & 2 & 0 & 0 & 4 \\
\hline Cocytius antaeus (Drury, 1773) & 0 & 0 & 0 & 0 & 0 & 1 & 0 & 1 & 2 \\
\hline Cocytius beelzebuth (Boisduval, 1875) & 0 & 1 & 0 & 0 & 0 & 0 & 0 & 0 & 1 \\
\hline Cocytius duponchel (Poey, 1832) & 1 & 1 & 0 & 0 & 0 & 0 & 0 & 0 & 2 \\
\hline Cocytius lucifer Rothschild \& Jordan, 1903 & 0 & 1 & 0 & 0 & 0 & 0 & 0 & 0 & 1 \\
\hline Enyo gorgon (Cramer, 1777) & 2 & 0 & 0 & 0 & 0 & 0 & 0 & 5 & 7 \\
\hline Enyo lugubris (Linnaeus, 1771) & 0 & 9 & 0 & 0 & 0 & 0 & 0 & 0 & 9 \\
\hline Enyo ocypete (Linnaeus, 1758) & 0 & 1 & 0 & 0 & 0 & 0 & 0 & 0 & 1 \\
\hline Erinnyis alope (Drury, 1773) & 3 & 14 & 0 & 1 & 0 & 1 & 0 & 0 & 19 \\
\hline Erinnyis crameri (Schaus, 1898) & 1 & 4 & 0 & 0 & 0 & 0 & 0 & 0 & 5 \\
\hline Erinnyis ello (Linnaeus, 1758) & 1 & 44 & 1 & 4 & 0 & 2 & 0 & 3 & 55 \\
\hline Erinnyis lassauxi (Boisduval, 1859) & 0 & 1 & 0 & 0 & 0 & 0 & 0 & 0 & 1 \\
\hline Erinnyis obscura obscura (Fabricius, 1775) & 0 & 0 & 0 & 1 & 0 & 0 & 0 & 0 & 1 \\
\hline Erinnyis oenotrus (Cramer, 1782) & 0 & 10 & 0 & 0 & 0 & 1 & 0 & 0 & 11 \\
\hline Eumorpha anchemolus (Cramer, 1780) & 0 & 1 & 0 & 0 & 0 & 1 & 0 & 0 & 2 \\
\hline Eumorpha labruscae labruscae (Linnaeus, 1758) & 0 & 7 & 0 & 0 & 1 & 0 & 0 & 0 & 8 \\
\hline Eumorpha obliqua (Rothschild \& Jordan, 1903) & 0 & 2 & 0 & 0 & 0 & 0 & 0 & 0 & 2 \\
\hline Eumorpha vitis (Linnaeus, 1758) & 0 & 0 & 0 & 0 & 0 & 1 & 1 & 0 & 2 \\
\hline Hemeroplanes longistriga (Rothsch. \& Jordan, 1903) & 0 & 0 & 0 & 0 & 1 & 0 & 0 & 0 & 1 \\
\hline Hemeroplanes omatus Rothschild, 1894 & 2 & 0 & 0 & 0 & 0 & 0 & 0 & 0 & 2 \\
\hline Manduca florestan (Cramer, 1782) & 0 & 16 & 1 & 0 & 0 & 4 & 1 & 0 & 22 \\
\hline Manduca grupo "diffissa" & 0 & 1 & 2 & 0 & 0 & 4 & 2 & 2 & 11 \\
\hline Manduca grupo "pellenia" & 0 & 19 & 0 & 1 & 2 & 5 & 3 & 0 & 30 \\
\hline Manduca hannibal (Cramer, 1779) & 0 & 0 & 0 & 0 & 0 & 3 & 0 & 0 & 3 \\
\hline Manduca incisa (Walker, 1856) & 0 & 0 & 0 & 1 & 0 & 0 & 1 & 0 & 2 \\
\hline Manduca rustica (Fabricius, 1775) & 0 & 0 & 0 & 0 & 1 & 17 & 1 & 0 & 19 \\
\hline Manduca sexta paphus (Cramer, 1779) & 0 & 0 & 0 & 0 & 0 & 5 & 1 & 0 & 6 \\
\hline Manduca sp. & 0 & 0 & 0 & 0 & 0 & 2 & 2 & 0 & 4 \\
\hline Neococytius cluentius (Cramer, 1775) & 0 & 0 & 0 & 0 & 0 & 1 & 0 & 0 & 1 \\
\hline Nyceryx continua (Walker, 1856) & 0 & 71 & 0 & 3 & 5 & 0 & 13 & 0 & 92 \\
\hline Nyceryx nictitans nictitans (Boisduval, 1875) & 0 & 0 & 0 & 2 & 3 & 0 & 3 & 0 & 8 \\
\hline Orecta lycidas (Boisduval, 1875) & 0 & 2 & 0 & 0 & 0 & 0 & 0 & 0 & 2 \\
\hline Pachylia ficus (Linnaeus, 1758) & 0 & 1 & 0 & 0 & 0 & 2 & 0 & 1 & 4 \\
\hline Pachylioides resumens (Walker, 1856) & 1 & 2 & 0 & 0 & 1 & 0 & 4 & 3 & 11 \\
\hline Protambulyx strigilis (Linnaeus, 1771) & 0 & 1 & 1 & 7 & 1 & 7 & 0 & 0 & 17 \\
\hline Xylophanes aglaor (Boisduval, 1875) & 0 & 1 & 0 & 0 & 0 & 0 & 0 & 0 & 1 \\
\hline Xylophanes anubus (Cramer, 1777) & 0 & 0 & 0 & 0 & 0 & 1 & 0 & 0 & 1 \\
\hline Xylophanes ceratomioides (Grote \& Robinson, 1867) & 2 & 1 & 2 & 5 & 0 & 0 & 0 & 0 & 10 \\
\hline Xylophanes chiron nechus (Cramer, 1777) & 1 & 16 & 1 & 0 & 0 & 0 & 0 & 1 & 19 \\
\hline Xylophanes hydrata Rothschild \& Jordan, 1903 & 0 & 0 & 0 & 0 & 0 & 0 & 0 & 1 & 1 \\
\hline Xylophanes indistincta Closs, 1915 & 0 & 32 & 0 & 0 & 0 & 0 & 1 & 0 & 33 \\
\hline Xylophanes isaon (Boisduval, 1875) & 1 & 17 & 0 & 0 & 0 & 0 & 0 & 0 & 18 \\
\hline X. porcus continentalis Rothsch. \& Jordan, 1903 & 0 & 11 & 0 & 1 & 0 & 0 & 0 & 0 & 12 \\
\hline Xylophanes pluto (Fabricius, 1777) & 0 & 0 & 0 & 0 & 0 & 5 & 0 & 1 & 6 \\
\hline Xylophanes schausi (Rothschild, 1894) & 0 & 1 & 0 & 0 & 0 & 0 & 0 & 0 & 1 \\
\hline Xylophanes tersa (Linnaeus, 1771) & 0 & 36 & 8 & 0 & 0 & 5 & 1 & 0 & 50 \\
\hline Xylophanes thyelia (Linnaeus, 1758) & 1 & 3 & 0 & 0 & 0 & 0 & 0 & 0 & 4 \\
\hline Xylophanes titana (Druce, 1878) & 0 & 14 & 0 & 0 & 1 & 1 & 0 & 0 & 16 \\
\hline Xylophanes tyndarus (Boisduval, 1875) & 0 & 3 & 0 & 1 & 0 & 0 & 0 & 0 & 4 \\
\hline Xylophanes xylobotes (Burmeister, 1878) & 0 & 10 & 1 & 1 & 0 & 0 & 0 & 0 & 12 \\
\hline Total de exemplare & 24 & 486 & 21 & 61 & 27 & 94 & 68 & 32 & 813 \\
\hline Total de espécies & 15 & 38 & 10 & 15 & 11 & 23 & 14 & 11 & 55 \\
\hline Total de espécies capturadas apenas no local & 1 & 10 & 0 & 1 & 1 & 3 & 0 & 1 & - \\
\hline
\end{tabular}


Tabela III. Espécies com maior número de exemplares capturados nas oito localidades, com o percentual em relação ao total capturado em cada localidade. Localidades em ordem decrescente da porcentagem da espécie mais abundante.

\begin{tabular}{|c|c|c|c|c|c|c|}
\hline Localidade & $\begin{array}{c}\text { Total de } \\
\text { exemplares }\end{array}$ & $\begin{array}{l}\text { Total de } \\
\text { espécies }\end{array}$ & Espécies & $\begin{array}{l}\text { Número de } \\
\text { exemplares }\end{array}$ & $\begin{array}{r}\text { Porcente } \\
(\%)\end{array}$ & agem \\
\hline \multirow[t]{2}{*}{ Guarapuava } & 68 & 14 & Adhemarius gannascus & 34 & 50,0 & \\
\hline & & & Nyceryx continua & 13 & 19,1 & 69,1 \\
\hline \multirow[t]{2}{*}{ Fênix } & 32 & 11 & Adhemarius gannascus & 13 & 40,6 & \\
\hline & & & Enyo gorgon & 5 & 15,6 & 56,2 \\
\hline \multirow[t]{2}{*}{ Colombo } & 21 & 10 & Xylophanes tersa & 8 & 38,1 & \\
\hline & & & Adhemarius gannascus & 3 & 14,3 & 52,4 \\
\hline \multirow[t]{2}{*}{ Ponta Grossa } & 61 & 15 & Adhemarius gannascus & 22 & 36,1 & \\
\hline & & & Adhemarius eurysthenes & 10 & 16,4 & 52,5 \\
\hline \multirow[t]{2}{*}{ Telêmaco Borba } & 27 & 11 & Adhemarius gannascus & 9 & 33,3 & \\
\hline & & & Nyceryx continua & 5 & 18,5 & 51,8 \\
\hline \multirow[t]{2}{*}{ Jundial do Sul } & 94 & 23 & Adhemarius gannascus & 22 & 23,4 & \\
\hline & & & Manduca rustica & 17 & 18,1 & 41,5 \\
\hline \multirow[t]{2}{*}{ Antonina } & 24 & 15 & Adhemarius gannascus & 4 & 16,7 & \\
\hline & & & Erinnys alope & 3 & 12,5 & 29,2 \\
\hline \multirow[t]{2}{*}{ São José dos Pinhais } & 486 & 38 & Nyceryx continua & 71 & 14,6 & \\
\hline & & & Adhemarius eurysthenes & 69 & 14,2 & 28,8 \\
\hline
\end{tabular}

Tabela IV. Número de espécies de Sphingidae capturadas a cada novilúnio (mais dois dias anteriores e dois posteriores), por localidade, e o número acumulado de espécies nominais capturadas, de outubro de 1986 a setembro de 1987.

\begin{tabular}{|c|c|c|c|c|c|c|c|c|c|c|c|c|c|}
\hline Novilúnio & 1 & 2 & 3 & 4 & 5 & 6 & 7 & 8 & 9 & 10 & 11 & 12 & 13 \\
\hline Localidade / Data & $03 / X$ & $02 / X 1$ & $01 /$ XII & $31 / X I 1$ & $29 / 1$ & $27 / 11$ & $29 / I I I$ & $27 / \mathrm{VV}$ & $27 N$ & $26 / \mathrm{VI}$ & $25 \mathrm{NII}$ & 24 NIII & $23 / 1 X$ \\
\hline Antonina & 1 & 1 & 3 & 1 & 2 & 4 & 5 & 0 & 1 & 0 & 2 & 2 & 0 \\
\hline São José Pinhais & 3 & 11 & 17 & 19 & 19 & 20 & 17 & 5 & 2 & 0 & 9 & 8 & 8 \\
\hline Colombo & 0 & 6 & 0 & 1 & 3 & 3 & 0 & 1 & 0 & 0 & 1 & 1 & 1 \\
\hline Ponta Grossa & 4 & 2 & 4 & 3 & 3 & 4 & 5 & 3 & 1 & 0 & 2 & 2 & 2 \\
\hline Telêmaco Borba & 3 & 4 & 1 & 2 & 2 & 0 & 3 & 1 & 2 & 0 & 0 & 1 & 2 \\
\hline Jundiai do Sul & 6 & 4 & 13 & 3 & 12 & 5 & 2 & 4 & 0 & 0 & 2 & 3 & 2 \\
\hline Guarapuava & 3 & 12 & 0 & 2 & 3 & 4 & 4 & 3 & 0 & 0 & 1 & 0 & 1 \\
\hline Fênix & 7 & 3 & 4 & 0 & 1 & 0 & 1 & 1 & 0 & 0 & 1 & 1 & 3 \\
\hline $\begin{array}{l}\text { Número acumulado de } \\
\text { espécies nominais }\end{array}$ & 18 & 33 & 39 & 42 & 43 & 48 & 50 & 52 & 52 & 52 & 53 & 54 & 55 \\
\hline
\end{tabular}

Nas demais localidades, houve um pequeno número de exemplares coletados para as diferentes espécies. Em locais com número mais elevado de exemplares, a captura foi mais uniforme ao longo do período, sem picos notáveis nos diferentes meses. Em maio e junho, em alguns locais, não houve captura de nenhum exemplar.

Em cada local, a soma dos exemplares de todas as espécies (Figs 2-9), indica que em Fênix, a maior abundância (9 exemplares) ocorreu em outubro; em Colombo (8), Telêmaco Borba (5) e Guarapuava (19), em novembro; em Jundiaí do Sul (22), em janeiro; e em Antonina (5), São José dos Pinhais (92) e Ponta Grossa (10), em março.

Revta bras. Zool. 16 (Supl. 2): 223 - 240, 1999 
2

ANTONINA

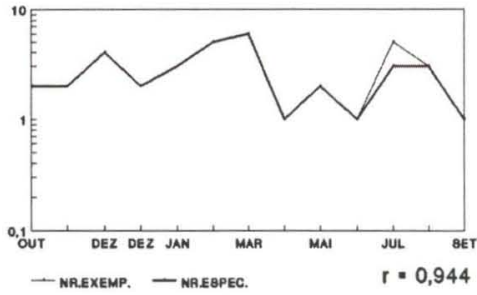

4

COLOMBO

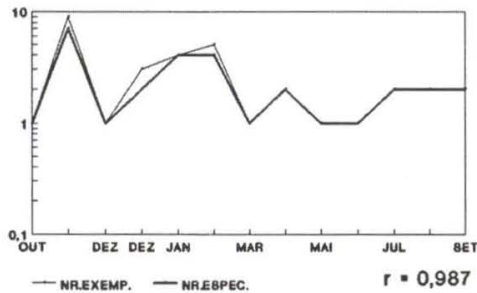

6

TELEMACO BORBA

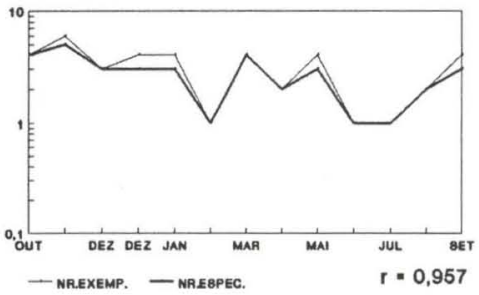

8

GUARAPUAVA

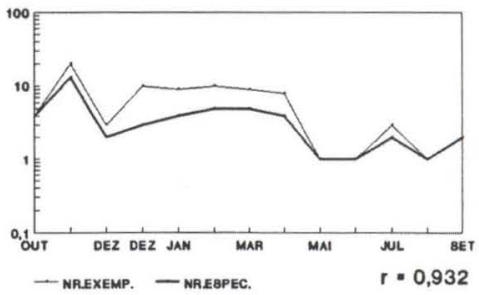

3

S.JOSE DOS PINHAIS

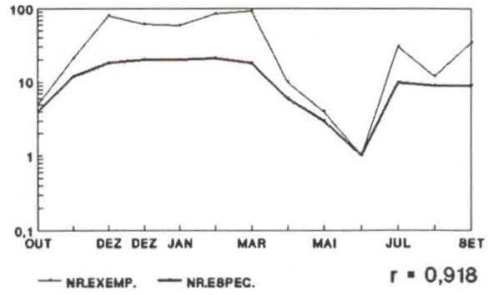

PONTA GROSSA

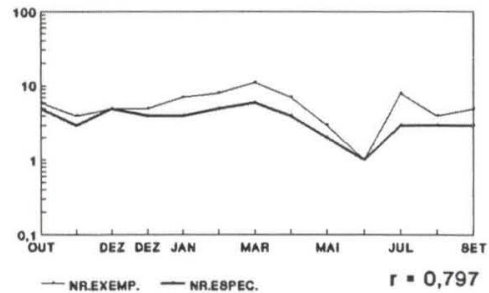

7

JUNDIAÍ DO SUL

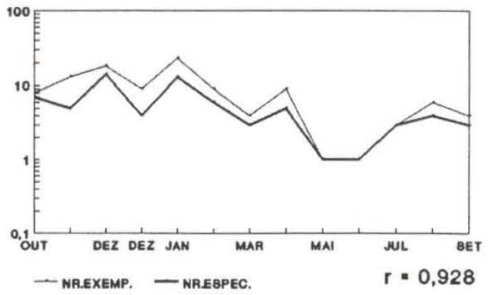

9

FÊNIX

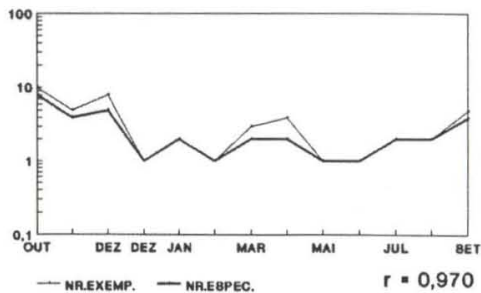

Figs 2-9. Gráfico de cada um dos locais de coleta, com número de exemplares (traço fino) e número de espécies (traço grosso) de Sphingidae, em valores logaritmos, capturadas a cada novilúnio. (r) Coeficiente de correção linear. 
Nas figuras 2 a 9 estão representados os valores relativos ao número de exemplares $(\mathrm{N})$ e de variedade de espécies $(\mathrm{S})$ capturados a cada novilúnio nas oito localidades, com o respectivo coeficiente de correlação linear. Os coeficientes mais altos foram os de Colombo $(0,987)$ e Fênix $(0,970)$, e o mais baixo, o de Ponta Grossa $(0,797)$.

Seis localidades tiveram os mais altos valores de abundância nos mesmos novilúnios em que houve a maior variedade de espécies capturadas, exceto as localidades de São José dos Pinhais e de Jundiaí do Sul.

CAPTURA MÉdIA DE SPHINGIDAE (número de exemplares dividido pelo número de coletas) (Tab. V)

A localidade de São José dos Pinhais foi a que apresentou o maior valor de captura média por novilúnio $(7,48)$, muito superior àquela de Jundiaí do Sul $(1,45)$ e a de Guarapuava $(1,05)$. Colombo apresentou o menor valor por novilúnio $(0,33)$.

Os maiores valores de captura média, em cada localidade, foram: em princípio de outubro em Fênix; em princípio de novembro em Colombo, Telêmaco e Guarapuava; em fins de janeiro em Jundiaí do Sul; e, em fins de março em Antonina, São José e Ponta Grossa.

Os valores referentes às médias por novilúnio englobando todas as localidades, comparado ao número total de espécies capturadas por novilúnio, em todas as localidades, apresentou um coeficiente de correlação linear igual a 0,841 .

Tabela V. Captura média de Sphingidae (número de exemplares/número de coletas), por localidade, no dia de novilúnio (mais dois dias anteriores e dois posteriores) de outubro de 1986 a setembro de 1987.

\begin{tabular}{|c|c|c|c|c|c|c|c|c|c|c|c|c|c|c|}
\hline Novilúnio & 1 & 2 & 3 & 4 & 5 & 6 & 7 & 8 & 9 & 10 & 11 & 12 & 13 & \multirow{2}{*}{$\begin{array}{r}\text { Média } \\
\text { - global }\end{array}$} \\
\hline Localidade / Data & $03 / x$ & $02 / X 1$ & $01 / X \mid 1$ & $31 / X I 1$ & $29 / 1$ & $27 / 11$ & $29 / I I I$ & $27 / I V$ & $27 N$ & $26 / \mathrm{NI}$ & $25 / \mathrm{NII}$ & $24 N I I I$ & $23 / 1 x$ & \\
\hline Antonina & 0,2 & 0,2 & 0,6 & 0,2 & 0,4 & 0,8 & 1,0 & 0,0 & 0,2 & 0,0 & 0,8 & 0,4 & 0,0 & 0,38 \\
\hline São José Pinhais & 0,8 & 4.0 & 16,0 & 12,2 & 11,6 & 17,0 & 18,4 & 1,8 & 0,8 & 0,0 & 5,8 & 2,2 & 6,6 & 7,48 \\
\hline Colombo & 0,0 & 2,7 & 0,0 & 0,4 & 0,6 & 0,8 & 0,0 & 0,2 & 0,0 & 0,0 & 0,2 & 0,2 & 0,2 & 0,33 \\
\hline Ponta Grossa & 1,0 & 0,6 & 0,8 & 0,8 & 1.2 & 1,4 & 2,0 & 1,2 & 0,2 & 0,0 & 1,4 & 0,6 & 0,8 & 0,94 \\
\hline Telẽmaco Borba & 0,6 & 1,0 & 0,4 & 0,6 & 0,6 & 0,0 & 0,6 & 0,2 & 0,6 & 0,0 & 0,0 & 0,2 & 0,6 & 0,42 \\
\hline Jundiai do Sul & 1,4 & 2,4 & 3,4 & 1,6 & 4,4 & 1,6 & 0,6 & 1,6 & 0,0 & 0,0 & 0,4 & 1,0 & 0,4 & 1,45 \\
\hline Guarapuava & 0,6 & 3,8 & 0,4 & 1,8 & 1,6 & 1,8 & 1,6 & 1,4 & 0,0 & 0,0 & 0,4 & 0,0 & 0,2 & 1,05 \\
\hline Fênix & 1,8 & 0,8 & 1,4 & 0,0 & 0,3 & 0,0 & 0,4 & 0,8 & 0,0 & 0,0 & 0,2 & 0,5 & 1,3 & 0,51 \\
\hline Média global & 0,8 & 1,9 & 2,9 & 2,2 & 2,6 & 2,9 & 3,1 & 0,9 & 0,2 & 0,0 & 1,2 & 0,6 & 1,3 & \\
\hline
\end{tabular}

\section{Análise numérica da captura média}

Análise de Agrupamento entre as 8 localidades baseada nos vetores dos valores de captura média de cada um dos 13 novilúnios, conforme tabela $\mathrm{V}$. (8 OTU's x 13 caracteres; coeficiente de semelhança por correlação linear; UPGMA) (Coeficiente de correlação cofenética $=0,877$ ) (Árvore I, Fig. 18).

Tendo como núcleo as localidades de Colombo e Guarapuava, as localidades de Telêmaco Borba, Jundiaí do Sul, mais Fênix, constituem um subconjunto, afastado do subconjunto formado por Ponta Grossa, mais o núcleo Antonina - São José dos Pinhais. 
10

\section{ANTONINA}

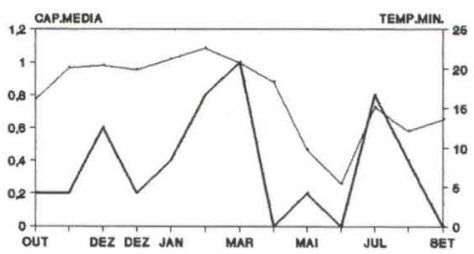

- CAP.MEdia - TEMP.Min.

$r=0,489$

$$
12
$$

COLOMBO

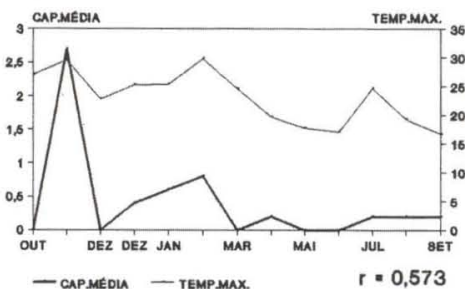

14

\section{TELÊMACO BORBA}

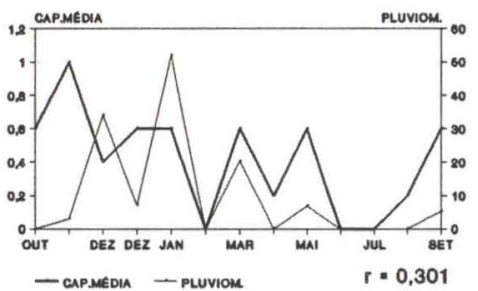

16

\section{GUARAPUAVA}

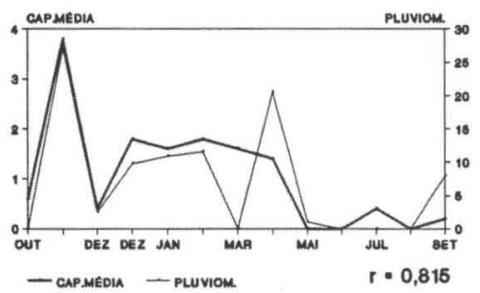

11

S.JOSE DOS PINHAIS

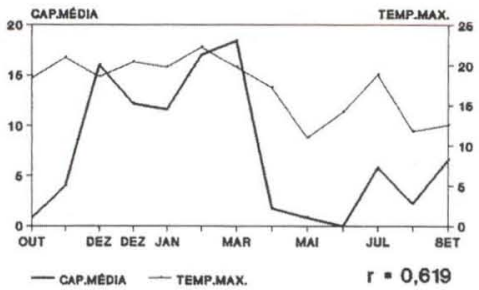

13 PONTA GROSSA

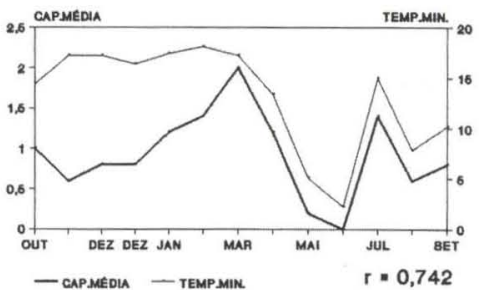

15 JUNDIAÍ DO SUL

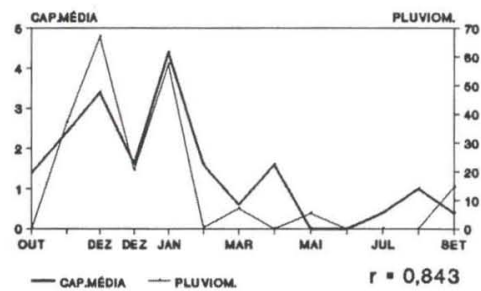

17

FENNIX

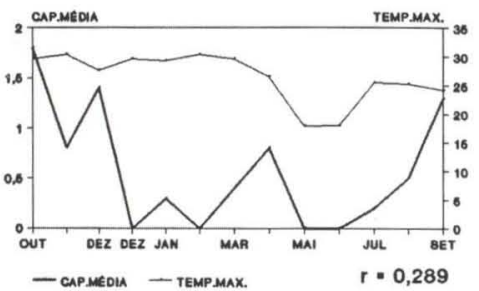

Figs 10-17. Gráfico de cada um dos locais de coleta com valor médio de exemplares de Sphingidae capturados por novilúnio (traço grosso) e os valores da variável meteorológica (traço fino) com a qual houve o maior coeficiente de correlação linear. 

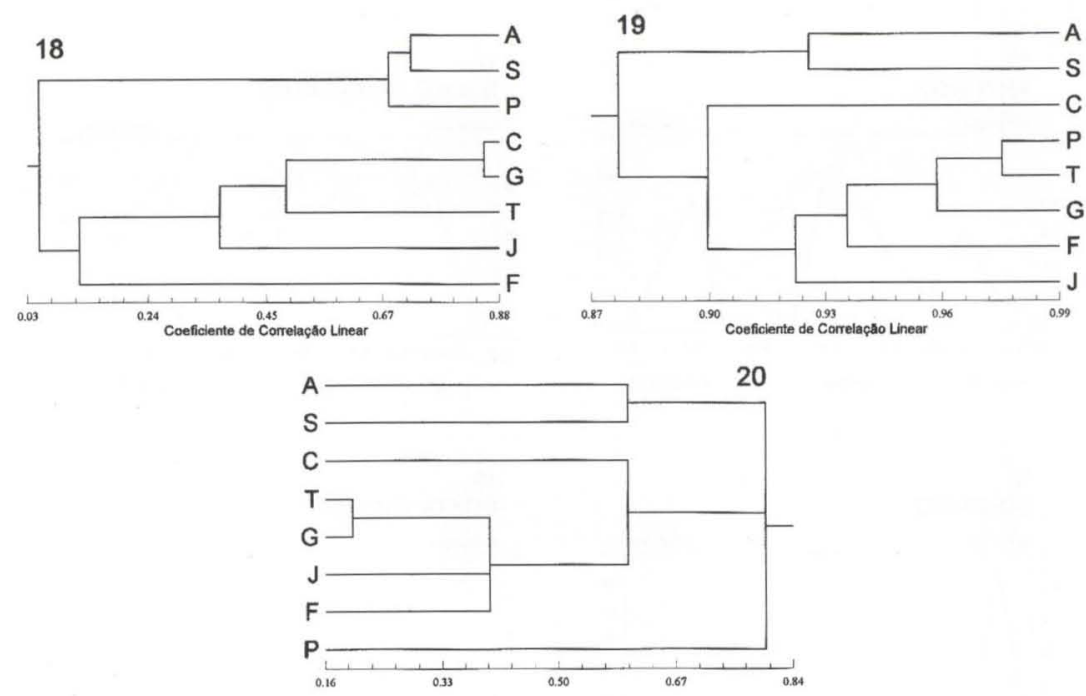

Figs 18-20. Análises de agrupamento. (18) Árvore I - locais de coleta x valores de captura média de exemplares de Sphingidae de cada um dos 13 novilúnios; (19) Árvore II - locais de coleta x valores de temperatura máxima nos 13 novilúnios; (20)Árvore III-Árvore de consenso entre Árvore I, de captura média de Sphingidae e Árvore II, com valores de temperatura. (A) Antonina, (C) Colombo, (F) Fênix, (G) Guarapuava, (J) Jundiaí do Sul, (P) Ponta Grossa, (S) São José dos Pinhais, (T) Telêmaco Borba.

\section{Captura média x clima}

Nas análises de consenso entre fenogramas obtidos com base em captura média por novilúnio, por localidade (Fig. 18) e diferentes fenogramas obtidos a partir de cada uma das variáveis meteorológicas, observou-se uma relação maior com aquela gerada a partir de dados de temperatura máxima (Fig. 19; 8 OTU's x 13 caracteres; coeficiente de semelhança por correlação linear; UPGMA; Coeficiente de correlação cofenética $=0,654)$, ou seja, a variação da temperatura nos 13 novilúnios resultou numa variação correspondente da captura. Na árvore de consenso (Fig. 20; pelo método de Stinebrickner, parâmetro de interseção $=0,5$; Índice de Colless $=0,667 ; 4$ subconjuntos) reconhecem-se os subconjuntos formados por Antonina e São José dos Pinhais; Telêmaco Borba e Guarapuava, ao qual se unem Jundiaí do Sul e Fênix, e ainda Colombo. Totalmente isolada a localidade de Ponta Grossa.

A análise por correlação linear entre os vetores com valores de captura média de cada novilúnio e os vetores com valores das variáveis meteorológicas (Tab. VI, Figs 10-17), apresentou, no conjunto, coeficientes baixos, indicando pouca correlação. Houve maior correlação entre captura média e: 1) temperatura máxima nas localidades de São José dos Pinhais $(0,619)$, Colombo $(0,573)$ e Fênix $(0,289) ; 2)$ temperatura mínima em Antonina $(0,489)$ e Ponta Grossa $(0,742) ; 3)$ e pluviometria em Telêmaco Borba $(0,301)$, Jundiaí do Sul $(0,843)$ e Guarapuava $(0,815)$. Os coeficientes de correlação com a umidade relativa do ar foram baixos em todas as localidades e negativos na maioria.

Revta bras. Zool. 16 (Supl. 2): 223 - 240, 1999 
Tabela VI. Coeficientes de Correlação Linear ( $r$ ) entre os dados de captura média de Sphingidae e as variáveis meteorológicas das oito localidades, nos novilúnios, de outubro de 1986 a setembro de 1987.

\begin{tabular}{lcccc}
\hline \multicolumn{1}{c}{ Localidade } & Temperatura máxima & Temperatura minima & Umidade relativa do ar & Pluviometria \\
\hline Antonina & 0,426 & 0,489 & $-0,002$ & 0,231 \\
Săo José Pinhais & 0,619 & - & $-0,189$ & 0,352 \\
Colombo & 0,573 & 0,341 & $-0,208$ & 0,534 \\
Ponta Grossa & 0,640 & 0,742 & $-0,130$ & $-0,062$ \\
Telêmaco Borba & 0,179 & 0,222 & $-0,014$ & 0,301 \\
Jundiai do Sul & 0,362 & 0,763 & 0,331 & 0,843 \\
Guarapuava & 0,613 & 0,641 & 0,035 & 0,815 \\
Fênix & 0,289 & 0,219 & $-0,043$ & 0,134 \\
\hline
\end{tabular}

\section{Discussão das relações entre a captura média e as variáveis meteoro-} lógicas (dados históricos e do período 1986/87, in MARINONI \& DUTRA 1991)

Observa-se que os valores de captura média a cada novilúnio se mostraram mais relacionados à temperatura (máxima e mínima), na maioria das localidades. Apenas Jundiaí do Sul e Guarapuava apresentaram mais alta correlação com a pluviometria (os maiores coeficientes de correlação dentre todos). As localidades de Fênix e Telêmaco Borba foram as que apresentaram os mais baixos coeficientes de correlação entre os valores de captura média e as variáveis meteorológicas.

A comparação entre o número total de exemplares capturados e os coeficientes de correlação linear indicam que em São José dos Pinhais, onde houve a maior abundância, o maior coeficiente (captura média x variável meteorológica) foi com a temperatura, enquanto que as duas localidades seguintes de maior abundância, foram Jundiaí do Sul e Guarapuava, onde o maior coeficiente foi com a pluviometria.

São José dos Pinhais apresentou o maior valor de captura média do total do período de captura $(7,48)$, cerca de 5 vezes superior à segunda média, a de Jundiaí do Sul $(1,45)$. A média mais baixa foi a de Antonina $(0,37)$. Comparando-se este valores às médias do período de coleta e às médias históricas de pluviosidade observa-se que São José dos Pinhais e Antonina são as que apresentaram as maiores precipitações de chuva e têm os valores extremos, maior e menor, respectivamente, de captura de exemplares.

\section{ÍNDICES DE DIVERSIDADE E DE DOMINÂNCIA/UNIFORMIDADE (=EVENESS) (Tab. VII) Índices de diversidade}

Foram calculados três índices de diversidade: de Brillouin, Shannon e $\alpha$ de Williams. A aplicação destes dois últimos índices, além do de Brillouin, julgado mais adequado para os dados coligidos através de armadilha luminosa, é decorrência do uso bastante comum dos mesmos, e que, de certa forma, possibilitam algumas análises comparativas.

Os índices de Brillouin e de Shannon, apesar de atribuírem valores diferentes às diversidades das localidades, mostram na análise relativa praticamente os mesmos resultados. Assim, São José dos Pinhais, Jundiaí do Sul e Antonina têm índices que, pela ordem, as posicionam como sendo as três localidades de maior diversidade. Guarapuava, Fênix e Colombo com os menores valores de diversidade. 
As duas localidades indicadas como de maior diversidade pelos índices HB e H', também foram as que apresentaram os maiores valores de $\mathrm{S}$ (variedade de espécies) e de $\mathrm{N}$ (abundância de exemplares).

$\mathrm{O}$ índice $\alpha$ de Williams apresentou seu maior valor para Antonina, muito superior ao das localidades de Jundiaí do Sul e São José dos Pinhais, que se seguem em ordem decrescente. Com as localidades de Guarapuava e Fênix sendo indicadas com as de menor diversidade.

Tabela VII. Diversidade e Uniformidade de Sphingidae. (S) Número de espécies; (N) número de indivíduos coletados; $(\mathrm{HB})$ indice de diversidade de Brillouin; $\left(\mathrm{H}^{\prime}\right)$ índice de diversidade de Shannon; (a) indice alfa de Williams; (H'E) uniformidade de Shannon; (UBP) indice de uniformidade de Berger \& Parker; (BP) índice de dominância de Berger \& Parker; (1/D) índice de uniformidade de Simpson.

\begin{tabular}{lcrrrrrrrr}
\hline \multicolumn{1}{c}{ Localidade } & $\mathrm{S}$ & $\mathrm{N}$ & $\mathrm{HB}$ & $\mathrm{H}^{\prime}$ & $\alpha$ & $\mathrm{H}^{\prime} \mathrm{E}$ & UBP & BP & $1 / \mathrm{D}$ \\
\hline Antonina & 15 & 24 & 0,851 & 2,584 & 17,113 & 0,954 & 5,988 & 0,167 & 21,231 \\
Săo José Pinhais & 38 & 486 & 1,192 & 2,867 & 9,650 & 0,788 & 6,849 & 0,146 & 12,963 \\
Colombo & 10 & 21 & 0,654 & 1,970 & 7,478 & 0,855 & 2,625 & 0,381 & 6,364 \\
Ponta Grossa & 15 & 61 & 0,785 & 2,086 & 6,355 & 0,770 & 2,770 & 0,361 & 5,773 \\
Telêmaco Borba & 11 & 27 & 0,704 & 2,040 & 6,921 & 0,851 & 3,003 & 0,333 & 6,882 \\
Jundiai do Sul & 23 & 94 & 0,997 & 2,620 & 9,713 & 0,836 & 4,274 & 0,234 & 9,779 \\
Guarapuava & 14 & 68 & 0,656 & 1,751 & 5,347 & 0,664 & 2,000 & 0,500 & 3,488 \\
Fênix & 11 & 32 & 0,678 & 1,922 & 5,926 & 0,802 & 2,463 & 0,406 & 5,221 \\
\hline
\end{tabular}

\section{Índices de dominância e uniformidade}

Os valores do índice de dominância de Berger \& Parker (BP) e seu inverso $(\mathrm{UBP}=1 / \mathrm{BP})$ apontam as localidades de São José dos Pinhais, Antonina e Jundiaí do Sul como as de maior uniformidade, em oposição às localidades de Fênix e Guarapuava, com os mais altos valores de dominância. Apesar do índice de Simpson (1/D) apresentar valores absolutos muito maiores que os de Berger \& Parker, os valores relativos para as oito localidades são mais semelhantes que os do índice de uniformidade de Shannon (H'E).

$\mathrm{O}$ ordenamento das localidades baseado na ordem crescente dos valores percentuais das duas espécies mais abundantes no local (Tab. III) mostra uma seqüência de localidades semelhante à obtida quando se utiliza o índice de Berger \& Parker, que tem seu cálculo apoiado na razão entre a espécie mais abundante pelo total dos exemplares de todas as espécies. Fica bem evidenciado que em São José dos Pinhais e Antonina há uma menor dominância de espécies em oposição a Fênix e Guarapuava com forte dominância de uma espécie.

\section{ESTRUTURA DAS COMUNIDADES}

Conforme procedimentos já indicados em MARINONI \& DUTRA (1996) e MARINONI et al. (1997), foram estabelecidas comparações, entre as localidades, apoiadas nos valores de abundância das espécies, independente do táxon.

\section{Resultados}

Foi feita uma Análise de agrupamento, representada pela Árvore IV (Fig. 21) (Coeficiente de correlação linear, UPGMA; coeficiente de correlação cofenética $=0,737)$. Foram estabelecidos três subconjuntos, um constituído por São José dos 
Pinhais e Jundiaí do Sul, ao qual se uniu Antonina; outro formado por dois núcleos, Telêmaco Borba - Colombo mais Fênix - Ponta Grossa; a estes subconjuntos unindo-se Guarapuava.

Estes resultados, produto da correlação entre os vetores definidos pela dominância entre as espécies, mostram que as localidades que se agruparam mais proximamente têm índices de uniformidade mais semelhantes, principalmente ao de Berger \& Parker (UBP).

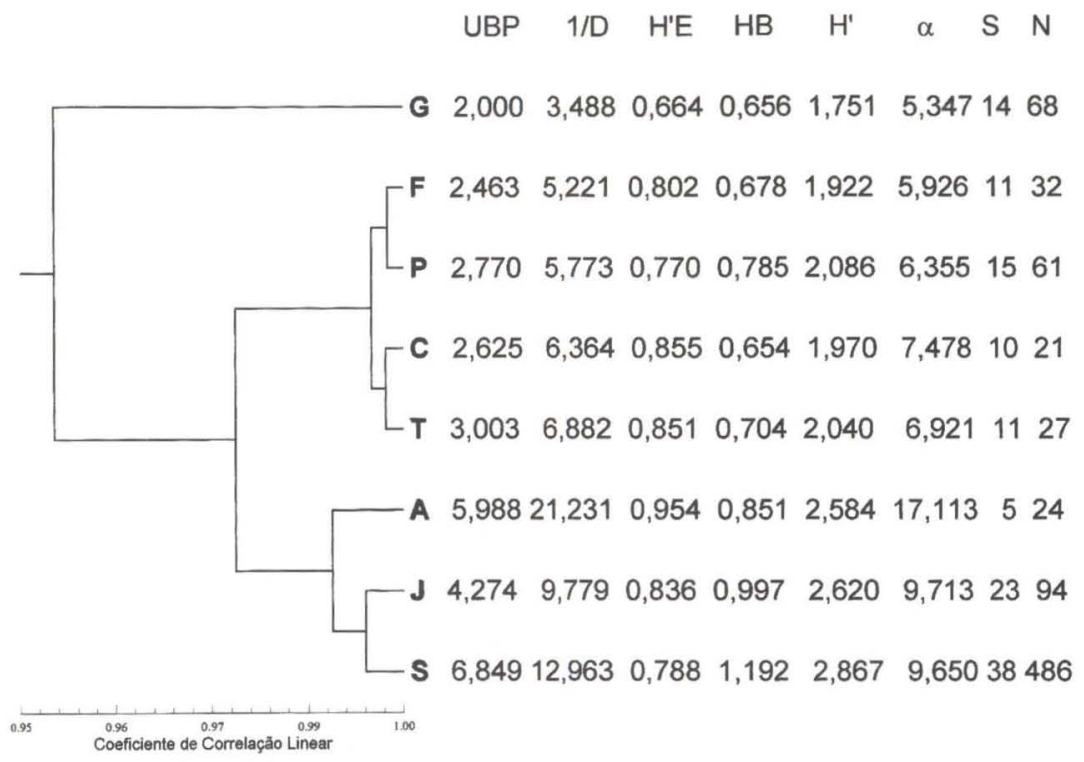

Fig. 21. Análise de agrupamento comparada com diferentes índices de diversidade e de uniformidade, com dados de Sphingidae. Árvore IV. Locais de coleta $x$ vetores formados por valores decrescentes do número de exemplares por espécie (ver texto - estrutura de comunidades). Colunas à direita correspondem aos indices de uniformidade de Berger \& Parker (UBP); de Simpson (1/D); de Shannon (H'E); aos índices de diversidade de Brillouin $(H B)$; de Shannon $(H) ; \alpha$ de Williams $(\alpha)$; e ao número de espécies $(S)$ e de exemplares $(N)$. (A) Antonina, (C) Colombo, (F) Fênix, (G) Guarapuava, (J) Jundiai do Sul, (P) Ponta Grossa, (S) São José dos Pinhais, (T) Telêmaco Borba.

Análises de consenso entre árvores de agrupamentos definidos com base nos padrões de estruturas de comunidades, aos pares de famílias (Sphingidae x Ctenuchidae; Sphingidae x Saturniidae), com dados e árvores de Ctenuchidae apresentados em MARINONI \& DUTRA (1996) e de Saturniidae, em MARINONI \& DUTRA (1997), mostraram maior semelhança entre Sphingidae e Ctenuchidae, conforme indicado pelas Árvores de Consenso (método de Stinebrickner; parâmetro de interseção $=0,5$ ). A maior semelhança entre Sphingidae e Ctenuchidae (Fig. 22; índice de Colless $=0,667$; índice de Mickevich $=0,583$ ) ocorreu ao agrupar em um subconjunto as localidades de São José dos Pinhais e Jundiaí do Sul; Ponta Grossa 
e Fênix, mais Colombo. Com as localidades de Antonina, Telêmaco Borba e Guarapuava mostrando poucas afinidades. Houve pouco consenso entre Sphingidae e Saturniidae (Fig. 23; índice de Colless =0,333; índice de Mickevich $=0,333$ ) tendo sido formado um único núcleo, indicando semelhança apenas entre as estruturas de comunidades de Ponta Grossa e Telêmaco Borba.
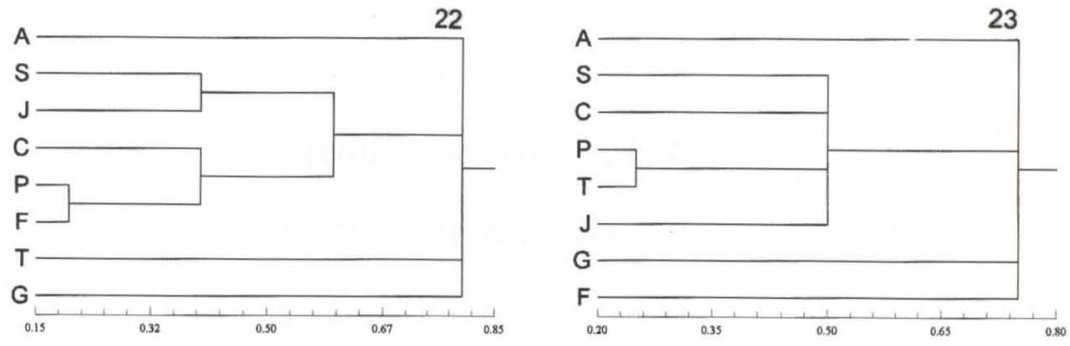

Figs 22-23. Análise de agrupamento. (22) Árvore V. Árvore de consenso entre agrupamentos de localidades em razão da semelhança entre estruturas de comunidades de Sphingidae (árv. IV, Fig. 21) e estruturas de comunidades de Ctenuchidae (MARINONI \& DUTRA 1996, Fig. 21). (A) Antonina, (C) Colombo, (F) Fênix, (G) Guarapuava, (J) Jundiaí do Sul, (P) Ponta Grossa, (S) São José dos Pinhais, (T) Telêmaco Borba. (23) Árvore VI. Árvore de consenso entre agrupamentos de localidades em razão da semelhança entre estruturas de comunidades de Sphingidae (árv. IV, Fig. 21) e estruturas de comunidades de Saturniidae (MARINONI et al. 1997, Fig. 21). (A) Antonina, (C) Colombo, (F) Fênix, (G) Guarapuava, (J) Jundiaí do Sul, (P) Ponta Grossa, (S) São José dos Pinhais, (T) Telêmaco Borba.

\section{Discussão sobre a variedade, número de exemplares capturados, diver- sidade, uniformidade $e$ as relações entre as estruturas das comunidades de Sphingidae}

A localidade que apresentou o maior número de espécies (38) e de exemplares capturados (486) foi São José dos Pinhais, como ocorreu com Saturniidae (MARINONI \& DUTRA 1997). Os valores encontrados foram muito superiores ao das demais localidades, tanto para espécies como para indivíduos. Poucas espécies e exemplares foram encontrados em Colombo e Telêmaco Borba.

Os resultados das três famílias estudadas Ctenuchidae, Saturniidae e Sphingidae, no que tange à riqueza de espécies e à abundância de indivíduos, mostram São José dos Pinhais como o local com os maiores valores de espécies e de indivíduos. Antonina e Fênix foram os locais em que se observaram os valores mais discrepantes entre as famílias, com alto número de espécies e indivíduos para os Ctenuchidae e baixo para os Saturniidae e Sphingidae. As demais localidades apresentaram relativo equilíbrio em todas as famílias estudadas, com Colombo sempre arrolada entre as localidades com mais baixo número de espécies e indivíduos. 


\section{Sphingidae $\mathbf{x}$ condições climáticas $\mathbf{x}$ condições florísticas}

Um dos maiores contrastes que se observa na análise dos Sphingidae está em Fênix. Como já observado anteriormente (MARINONI \& DUTRA 1991, 1996; MARINONI et al. 1997) Fênix é uma localidade rica em espécies vegetais, com clima quente e úmido e onde houve a maior coleta de Lepidoptera dentre todas as localidades estudadas. No entanto, foi onde se registrou, ao lado de Colombo, a menor variedade de espécies e a menor abundância de exemplares. Estes valores seriam semelhantes aos encontrados para os Saturniidae, na mesma localidade, não fosse ter havido para essa família uma maior captura de indivíduos, porém creditada à forte abundância de uma única espécie

De um modo geral, a variedade de espécies de Sphingidae nas demais localidades foi pequena quando comparada à de Saturniidae. Jundiaí do Sul e Antonina, locais com condições florísticas e climáticas que as aproximam de Fênix, foram também relativamente pobres em variedade e em abundância. Igualaram-se a todas as demais regiões, que sendo mais frias condicionaram uma captura menor de espécies e de indivíduos de Insecta (MARINONI \& DUTRA 1991), como Colombo, Ponta Grossa e Telêmaco Borba.

O estudo dos Sphingidae, de forma diferente das famílias Saturniidae e Ctenuchidae, não permitiu evidenciar relações mais estreitas de nenhum dos parâmetros ecológicos (riqueza de espécies, abundância de indivíduos, índices de diversidade e de uniformidade) às variáveis meteorológicas. Locais com mais baixa temperatura média apresentaram valores altos (São José dos Pinhais) e baixos (Colombo) de abundância de indivíduos e de riqueza de espécies, tanto quanto locais com mais alta temperatura média apresentaram valores altos (Jundiaí do Sul) e baixos (Antonina e Fênix). O mesmo ocorreu quando se avaliam os índices de diversidade e de uniformidade.

\section{OUtRos LEVANTAMENTOS DA FAUNA DE SPHINGIDAE}

Dois dos trabalhos, dentre aqueles que analisaram a fauna de Sphingidae, foram produto de estudo em áreas muito próximas à de São José dos Pinhais: LAROCA \& MiELKE (1975) e LAROCA et al. (1989). LAROCA \& MielKe (1975) em coleta noturna durante um ano, no pano, em Marumbi, Morretes, Paraná (510 metros de altitude) obtiveram 54 espécies e 1346 indivíduos; e LAROCA et al. (1989), com a mesma metodologia, coletaram 47 espécies e 497 indivíduos em Banhado, Quatro Barras, Paraná (830 m de altitude). No levantamento em São José dos Pinhais, distante aproximadamente $15 \mathrm{~km}$ ao sul daquelas localidades, foram coletados 38 espécies e 486 indivíduos. É possível constatar uma semelhança maior entre os dados de riqueza de espécies entre Marumbí e Banhado, e deste local com o São José dos Pinhais. Houve um decréscimo bastante acentuado da abundância de indivíduos de Marumbí para Banhado e São José dos Pinhais, que pode ter sido uma conseqüência natural pelo fato de Banhado e São José dos Pinhais situarem-se em áreas de maior altitude, onde as temperaturas médias são mais baixas; condição meteorológica que, como já observado por MARINONI \& DUTRA (1991), tem condicionado uma abundância menor de Insecta, de um modo geral, em diversas localidades paranaenses. 
Quando comparam-se as estruturas das comunidades de Sphingidae de São José dos Pinhais, Banhado e Marumbi (Tab. VIII), com base nas espécies nominais e seus níveis de dominância, listadas em ordem decrescente, tomadas para cada uma das localidades, vê-se que a correlação maior é entre Banhado e Marumbí $(r=0,573)$. A estrutura de São José dos Pinhais é mais semelhante à de Banhado $(r=-0,009)$ que à de Marumbi $(r=-0,527)$, possivelmente produto da maior semelhança na altitude. As relações mais estreitas entre Banhado e Marumbi do que entre Banhado e São José dos Pinhais contrastam com as condições fitogeográficas destes dois últimos locais, indicadas como semelhantes já que se situam em área de transição entre Floresta Ombrófila Densa Montana e Floresta Ombrófila Mista Montana, conforme classificação de Veloso \& Góes (LAROCA et al. 1989; MARINONI \& DUTRA 1991).

Os índices de uniformidade de Berger \& Parker para Marumbi $(3,542)$ e Banhado $(6,236)$ (calculados sobre os dados de abundância indicados nos citados trabalhos) comparados ao de São José dos Pinhais $(6,849)$ permitem indicar uma maior semelhança entre as duas últimas localidades.

Tabela VIII. (A) Lista de espécies das localidades de São José dos Pinhais (SJ), Banhado $(B A)^{\star}$ (Quatro Barras) e Marumbi (MA)* (Morretes), no Estado do Paraná, com número de ordem de dominância das primeiras seis espécies em cada local. Em cada localidade também estão arroladas as espécies classificadas dentre as seis mais abundantes em cada uma das outras duas localidades. (+) não arrolada entre as mais abundantes; (-) não citada para o local; (B) as onze espécies de cada local arroladas por ordem de abundância dentro desta análise. $\left(^{*}\right)$ In LAROCA et al. (1989).

\begin{tabular}{|c|c|c|c|c|c|c|}
\hline \multirow{2}{*}{ Espécie } & \multicolumn{3}{|c|}{ A } & \multicolumn{3}{|c|}{ B } \\
\hline & SJ & $\mathrm{BA}$ & MA & SJ & $\mathrm{BA}$ & MA \\
\hline Nyceryx continua & 1 & 8 & + & 1 & 7 & 10 \\
\hline Adhemarius eurysthenes & 2 & 6 & 13 & 2 & 6 & 8 \\
\hline Adhemanius gannascus & 3 & 12 & 20 & 3 & 9 & 9 \\
\hline Erinnyis ello & 4 & 2 & 1 & 4 & 2 & 1 \\
\hline Xylophanes tersa & 5 & 1 & 11 & 5 & 1 & 7 \\
\hline Xylophanes indistincta & 6 & - & - & 6 & 11 & 11 \\
\hline Erinnyis oenotrus & 15 & 3 & 3 & 11 & 3 & 3 \\
\hline Xylophanes chiron & 9 & 4 & 2 & 8 & 4 & 2 \\
\hline Manduca grupo "pellenia" & 7 & 5 & 5 & 7 & 5 & 5 \\
\hline Manduca florestan & 10 & 22 & 4 & 9 & 10 & 4 \\
\hline Agrius cingulatus & 13 & 11 & 6 & 10 & 8 & 6 \\
\hline
\end{tabular}

Coeficiente de correlação linear, com valores de $\mathrm{B}$ : entre $\mathrm{SJ} \times \mathrm{BA}=-0,009$; entre $\mathrm{SJ} \times \mathrm{MA}=$ $-0,527$; entre $B A \times M A=0,573$.

Os dados de riqueza de espécies e de abundância de exemplares dos outros levantamentos em áreas diferentes parecem coerentes com os obtidos no Estado do Paraná, apesar de terem se desenvolvido durante períodos mais longos ou mais curtos.

SEIFERT (1974), em Turrialba, Costa Rica, coletou 66 espécies e 565 indivíduos, durante cinco meses. Com o índice de Berger \& Parker igual a 3,767 (cálculo a partir de dados citados no trabalho), inferior aos de São José dos Pinhais, Antonina e Jundiaí do Sul. Tendo como espécies dominantes: Erinnyis ello, Adhemarius gannascus, Erinnyis oenotrus, Agrius cingulatus. As duas primeiras também dominantes no estado do Paraná. 
Coelho et al. (1979), em Piracicaba, São Paulo, coletaram 17 espécies e 646 exemplares, durante cinco anos. Com o índice de Berger \& Parker igual a 4,395 (cálculo partir de dados citados no trabalho), inferior aos de São José dos Pinhais e Antonina e pouco superior ao de Jundiai do Sul. Foram arroladas como espécies dominantes: Erinnyis ello, Xylophanes tersa, Erinnyis oenotrus, Calliomina parce e Pachyliodes resumens, as duas primeiras também dominantes no Paraná.

FERREIRA et al. (1986), em Viçosa, Minas Gerais, coletaram 35 espécies durante dois anos. Dentre as espécies arroladas entre as dominantes, Erinnyis ello e Xylophanes tersa também o são no Paraná.

Estes dados confirmam a larga distribuição e abundância de algumas espécies, como Erinnyis ello, E.oenotrus, Adhemarius gannascus, Xylophanes tersa, Agrius cingulatus. Da mesma forma, os valores do índice de Berger \& Parker, ainda que tomados a partir de dados obtidos de formas diversas (coleta e período), indicam uma provável situação de maior uniformidade da fauna de Sphingidae nas localidades de Banhado(Quatro Barras) e São José dos Pinhais, no alto da Serra do Mar, no Paraná, superiores também aos índices das sete outras localidades estudadas no Estado.

\section{CONSIDERAÇÕES FINAIS}

O levantamento da fauna de Sphingidae, em oito localidades do Estado do Paraná, através de armadilha luminosa, durante o período de um ano, indicou que:

- houve a captura de 813 exemplares, de 55 espécies; São José dos Pinhais foi a localidade onde se constatou maior abundância e riqueza de espécies (486:38) e Colombo, menor abundância e riqueza de espécies (21:10);

- o percentual acumulado de espécies capturadas nos três primeiros novilúnios $(71 \%)$ foi superior aos das famílias Ctenuchidae (66\%) e Saturniidae (65\%);

- Adhemarius gannascus foi capturada em todas as oito localidades e só não foi a mais abundante em Colombo e em São José dos Pinhais. As outras espécies mais abundantes foram Nyceryx continua, Adhemarius eurysthenes, Erinnyis ello, Xylophanes tersa e $X$. indistincta;

- São José dos Pinhais foi o local onde houve a maior captura média de indivíduos e Colombo, a menor;

- as variações nos valores de captura média estiveram mais relacionados às variações de temperatura na maioria das localidades, e aos valores de pluviometria em Jundiaí do Sul e Guarapuava;

- São José dos Pinhais e Jundiaí do Sul foram as duas localidades com maiores índices de diversidade (de Brillouin e de Shannon); e São José dos Pinhais e Antonina foram onde se observaram os maiores índices de uniformidade (de Berger \& Parker e de Simpson);

- não foi possivel estabelecer uma relação entre riqueza de espécie e abundância de indivíduos das localidades com qualquer uma das variáveis meteorológicas;

- as localidades que mostraram padrões semelhantes de estrutura de comunidades foram 1) São José dos Pinhais, Jundiaí do Sul e Antonina, 2) Colombo, Telêmaco Borba, Fênix e Ponta Grossa, e bem mais diferenciada, 3) Guarapuava; estas mesmas relações foram obtidas quando se analisam os índices de uniformidade 
ou ordenando as localidades com base no percentual das duas espécies mais abundantes.

AGRADECIMENTOS. Reiteramos os nossos agradecimentos contidos nos trabalhos anteriores que envolveram o estudo do material coletado pelo PROFAUPAR. Decorridos mais de dez anos do levantamento, fica cada vez mais nítido o significado do esforço despendido por todos aqueles que trabalharam de forma árdua e diuturna na coleta e triagem do material, criando uma fonte de informação entomológica até então inexistente para o estado do Paraná.

\section{REFERÊNCIAS BIBLIOGRÁFICAS}

BIEZANKo, C.M. 1948. Sphingidae de Pelotas e seus arredores. Contribuição ao conhecimento da fisiografia do Rio Grande do Sul. Pelotas, Edição do Autor, $8 \mathrm{p}$.

CarCasson, R.H. \& J.B. HePpner. 1996. Sphingoidea, Sphingidae, p.50-62. In: J.B. Heppner (Ed.) Atlas of Neotropical Lepidoptera, Checklist. Part 4B. Drepanoidea, Bombycoidea, Sphingoidea. Gainesville, Association for Tropical Lepidoptera, Scientific Publishers, 87p.

Coelho, I.P.; S. Silveira Neto; J.F.S. Dias; L.C. Forti; E.F. Chagas \& F.M. LARA. 1979. Fenologia e análise faunística da família Sphingidae (Lepidoptera), através de levantamentos com armadilha luminosa em Piracicaba-SP. An. Soc. Entomol. Brasil 8: 295-307.

FERREIRA, P.S.F.; D.S. MARTINS; N. HÜBNER. 1986. Levantamento, flutuação e análise entomofaunística em mata remanescente da Zona da Mata, Viçosa, Minas Gerais. I. Sphingidae: Lepidoptera. Rev. Ceres 33: 516-527.

LAROCA, S. \& O.H.H. MIELKE. 1975. Ensaios sobre ecologia de comunidade em Sphingidae na Serra do Mar, Paraná, Brasil (Lepidoptera). Rev. Brasil. Biol. 35: 1-19.

LAROCA, S.; V.O. BeCKER; F.C.V. ZANELlA. 1989. Diversidade, abundância relativa e fenologia de Sphingidae (Lepidoptera) na Serra do Mar (Quatro Barras, PR), sul do Brasil. Acta Biol. Paranaense, Curitiba, 18: 13-53 [1990]. MARINONI, R.C. \& R.R.C. DUTRA. 1991. Levantamento da Fauna Entomológica no Estado do Paraná. I. Introdução. Situações climática e florística de oito pontos de coleta. Dados faunísticos de agosto de 1986 a julho de 1987. Revta bras. Zool. 8: 31-73 [1993].

1996. Levantamento da Fauna Entomológica no Estado do Paraná. II. Ctenuchidae (Lepidoptera). Revta bras. Zool. 13 (2): 435-461.

MARINONI, R.C.; R.R.C. DutRA; M.M. CASAGRANDE. 1997. Levantamento da Fauna Entomológica no Estado do Paraná. II. Saturniidae (Lepidoptera). Revta bras. Zool. 14 (2): 473-495.

RoHLF, F.J. 1989. NTSYS-PC. Numerical Taxonomy and Multivariate Analysis System. New York, Exeter Publ. Ltd., VI+187p.

SEIFERT, R.P. 1974. The Sphingidae of Turrialba, Costa Rica. Jour. N.Y. Entomol. Soc. 82: $45-56$.

YouNG, A.M. 1972. Notes on a community ecology of adult sphinx moths in Costa Rica lowland tropical rain forest. Caribbean Jour. Science 12: 151-163. 